\title{
Research Article \\ Role of Delay on Planktonic Ecosystem in the Presence of a Toxic Producing Phytoplankton
}

\author{
Swati Khare, ${ }^{1}$ O. P. Misra, ${ }^{1}$ Chhatrapal Singh, ${ }^{1}$ and Joydip Dhar ${ }^{2}$ \\ ${ }^{1}$ School of Mathematics and Allied Sciences, Jiwaji University, MP Gwalior 474011, India \\ ${ }^{2}$ Department of Applied Sciences, ABV-Indian Institute of Information Technology and Management, \\ MP Gwalior 474010, India \\ Correspondence should be addressed to Swati Khare, swati9482@gmail.com
}

Received 11 May 2011; Accepted 25 July 2011

Academic Editor: Gershon Wolansky

Copyright @ 2011 Swati Khare et al. This is an open access article distributed under the Creative Commons Attribution License, which permits unrestricted use, distribution, and reproduction in any medium, provided the original work is properly cited.

\begin{abstract}
A mathematical model is proposed to study the role of distributed delay on plankton ecosystem in the presence of a toxic producing phytoplankton. The model includes three state variables, namely, nutrient concentration, phytoplankton biomass, and zooplankton biomass. The release of toxic substance by phytoplankton species reduces the growth of zooplankton and this plays an important role in plankton dynamics. In this paper, we introduce a delay (time-lag) in the digestion of nutrient by phytoplankton. The stability analysis of all the feasible equilibria are studied and the existence of Hopf-bifurcation for the interior equilibrium of the system is explored. From the above analysis, we observe that the supply rate of nutrient and delay parameter play important role in changing the dynamical behaviour of the underlying system. Further, we have derived the explicit algorithm which determines the direction and the stability of Hopf-bifurcation solution. Finally, numerical simulation is carried out to support the theoretical result.
\end{abstract}

\section{Introduction}

The study of plankton system is an important area of research in marine ecology. Phytoplankton perform great service for earth. They provide food for marine life, oxygen for human being and also absorb half of the carbon dioxide which may be contributing to the global warming [1]. The dynamics of rapid (massive) increase or almost equal decrease of phytoplankton population is a common feature in marine plankton ecology and known as bloom. Blooms of red tide produce chemical toxin, a type of paralytic poison which can be harmful to zooplankton, fin fish, shellfish, fish, birds, marine mammals, and humans also. Some species, such as the dinoflagellate Alexandrium tamarense and the diatom Pseudonitzschia australis [2] produce potent toxins which are liberated into the water before they are eaten, and they may well affect zooplankton when they are in water. It is now 
well established that a significant number of phytoplankton species produce toxin, such as Pseudo-nitzschia, Gambierdiscus toxicus, Prorocentrum, Ostrepsis, Coolia monotis, Thecadinium, Amphidinium carterae, Dinophysis, Gymnodinium breve, Alexandrium, Gymodinium catenatum, Pyrodinium bahamense, Pfiesteria piscicida, Chrysochromulina polylepis, Prymnesium patelliferum, and P. paroum [3,4]. Reduction of grazing pressure due to toxin is an important phenomena in plankton ecology $[5,6]$. In aquatic system, toxin-producing phytoplankton may act as controlling factor in the phytoplankton-zooplankton interaction dynamics. Efforts have been made to study the role of toxin producing phytoplankton on the phytoplankton-zooplankton dynamics [7-9]. Toxicity may be the strong mediator of zooplankton feeding rate as shown by field studies $[4,10]$ and laboratory study [11].

Many researchers have been showing keen interest to investigate the direction and stability of Hopf-bifurcation of the system as refer their in $[12,13]$.

Our current study is motivated by $[7,8,14]$, who have considered nutrient interaction with phytoplankton, phytoplankton interaction with zooplankton. The study of interacting species system with nutrient cycling which contributes to the growth of nutrient is carried out in $[9,14,15]$. A model can be more realistic if a delay effect (or time-lag) is being considered in the conversion from one species to another species [15-17]. The prey-predator systems with time delay are deeply considered by $[18,19]$ and many researchers have used distributed delay in their models $[15,16]$.

\section{Mathematical Model Formulation}

Let $N(t)$ denote the concentration of nutrient at time $t, x(t)$ denotes the biomass of toxic producing phytoplankton in the habitat that are partially harmful to zooplankton biomass, $y(t)$. We assume a constant supply rate of nutrient (i.e., $N_{0}$ ) in the system. The loss of nutrient due to leaching is assumed to be given by the term $a N$. We take $a_{1}$ as the growth rate of phytoplankton biomass, $w$ as the rate of predation of phytoplankton by zooplankton, and $w_{1}$ denotes the corresponding conversion rate of zooplankton. The phytoplankton and zooplankton interaction is assumed to follow the Holling type-II functional response [20, 21] with $D$ as half saturation constant. Again, the specific rate of nutrient uptake by per unit biomass of phytoplankton in unit time is considered to be $b N$ and depletion of zooplankton biomass due to toxin-producing phytoplankton is given by the term $c_{1} x y$. We further assume that phytoplankton and zooplankton biomass deplete due to natural mortality at the rate of $b_{1}$ and $a_{2}$, respectively, and $k$ is the fraction of dead phytoplankton biomass that is being recycled back to the nutrient pool. With these assumption and notations, the resultant dynamics of the system under consideration is given by the following set of differential equations [14]:

$$
\begin{aligned}
\frac{d N}{d t} & =N_{0}-a N-b N x+k b_{1} x, \\
\frac{d x}{d t} & =a_{1} N x-b_{1} x-\frac{w x y}{D+x} \\
\frac{d y}{d t} & =\frac{w_{1} x y}{D+x}-a_{2} y-c_{1} x y
\end{aligned}
$$

with nonnegative initial conditions $N(0), x(0), y(0)>0$. The above system of equations can be nondimensionalised using the relations $x_{1}=N / D, x_{2}=x / D, x_{3}=y / D, \tau=a t$, and 
introducing the new parameters $\alpha=N_{0} / a D, \beta=b D / a, \gamma=b_{1} / a, \beta_{1}=a_{1} D / a, \mu=w / a$, $\mu_{1}=w_{1} / a, \xi=a_{2} / a$, and $\xi_{1}=c_{1} D / a$. The non-dimensionalised equations of the above system (2.1) are as follows in which we have replaced $\tau$ by $t$ and get

$$
\begin{aligned}
& \frac{d x_{1}}{d t}=\alpha-x_{1}-\beta x_{1} x_{2}+k \gamma x_{2} \\
& \frac{d x_{2}}{d t}=\beta_{1} x_{1} x_{2}-\gamma x_{2}-\frac{\mu x_{2} x_{3}}{1+x_{2}} \\
& \frac{d x_{3}}{d t}=\frac{\mu_{1} x_{2} x_{3}}{1+x_{2}}-\xi x_{3}-\xi x_{2} x_{3}
\end{aligned}
$$

with initial conditions: $x_{1}(0)>0, x_{2}(0)>0, x_{3}(0)>0$, where, $\alpha, \beta, \gamma, \beta_{1}, \mu, \mu_{1}, \xi, \xi$, and $0<k<1$ are positive constants.

In this paper, our mathematical model is an extension of the system (2.2) which is studied earlier [14]. Now, we have introduced distributed delay in the digestion of nutrient by phytoplankton. The system (2.2) can be written as

$$
\begin{gathered}
\frac{d x_{1}}{d t}=\alpha-x_{1}-\beta x_{1} x_{2}+k \gamma x_{2}, \\
\frac{d x_{2}}{d t}=\beta_{1} x_{2} \int_{-\infty}^{t} \alpha_{1} \exp \left(-\alpha_{1}(t-s)\right) f(s) d s-\gamma x_{2}-\frac{\mu x_{2} x_{3}}{1+x_{2}},
\end{gathered}
$$

where $f(s)=f\left(x_{1}\right)=x_{1}(s), \alpha_{1}$ is delay parameter,

$$
\frac{d x_{3}}{d t}=\frac{\mu_{1} x_{2} x_{3}}{1+x_{2}}-\xi x_{3}-\xi_{1} x_{2} x_{3}
$$

putting

$$
R(t)=\int_{-\infty}^{t} \alpha_{1} \exp \left(-\alpha_{1}(t-s)\right) f(s) d s
$$

The above system of delay differential equation can be written as

$$
\begin{gathered}
\frac{d x_{1}}{d t}=\alpha-x_{1}-\beta x_{1} x_{2}+k \gamma x_{2} \\
\frac{d x_{2}}{d t}=\beta_{1} x_{2} R-\gamma x_{2}-\frac{\mu x_{2} x_{3}}{1+x_{2}} \\
\frac{d x_{3}}{d t}=\frac{\mu_{1} x_{2} x_{3}}{1+x_{2}}-\xi x_{3}-\xi x_{2} x_{3} \\
\frac{d R}{d t}=\alpha_{1}\left(x_{1}-R\right)
\end{gathered}
$$

with the additional initial condition: $R(0)>0$. 


\section{Boundedness and Equilibria of the System}

In this section, we will establish that the system (2.6) is bounded. We begin with the following lemma.

Lemma 3.1. The system (2.6) is uniformly bounded in $\Omega_{1}$, where

$$
\Omega_{1}=\left\{\left(x_{1}, x_{2}, x_{3}, R\right): 0 \leq x_{1}(t)+x_{2}(t)+x_{3}(t)+R(t) \leq \frac{\alpha}{\eta_{1}}\right\}
$$

$\eta_{1}=\min \left\{(1-\alpha), \gamma\left(1-k \beta_{1} / \beta\right), \xi, \alpha_{1}\right\}$

Proof. Let us consider a time dependent function:

$$
W_{1}(t)=x_{1}(t)+\frac{\beta}{\beta_{1}} x_{2}(t)+\frac{\beta \mu}{\beta_{1} \mu_{1}} x_{3}(t)+R(t)
$$

Clearly,

$$
\frac{d W_{1}}{d t}=\frac{d x_{1}}{d t}+\frac{\beta}{\beta_{1}} \frac{d x_{2}}{d t}+\frac{\beta \mu}{\beta_{1} \mu_{1}} \frac{d x_{3}}{d t}+\frac{d R}{d t}
$$

Using (2.6) in the above expression we obtain

$$
\begin{aligned}
\frac{d W_{1}}{d t}= & {\left[\alpha-x_{1}-\beta x_{1} x_{2}+k \gamma x_{2}\right]+\frac{\beta}{\beta_{1}}\left[\beta_{1} x_{2} R-\gamma x_{2}-\frac{\mu x_{2} x_{3}}{1+x_{2}}\right] } \\
& +\frac{\mu \beta}{\mu_{1} \beta_{1}}\left[\frac{\mu_{1} x_{2} x_{3}}{1+x_{2}}-\xi x_{3}-\xi 1 x_{2} x_{3}\right]+\left[\alpha_{1}\left(x_{1}-R\right)\right] \\
= & \alpha-\left(1-\alpha_{1}\right) x_{1}-\gamma\left(1-k \frac{\beta_{1}}{\beta}\right) \frac{\beta}{\beta_{1}} x_{2}-\xi \frac{\mu \beta}{\mu_{1} \beta_{1}} x_{3}-\xi \frac{\mu \beta}{\mu_{1} \beta_{1}} x_{2} x_{3}-\alpha_{1} R+\left(R-x_{1}\right) \beta x_{2} \\
\leq & \alpha-\eta_{1} W_{1}(t)+\left(R-x_{1}\right) \beta x_{2}
\end{aligned}
$$

where $\eta_{1}$ is chosen as the minimum of $\left\{\left(1-\alpha_{1}\right), \gamma\left(1-k \beta_{1} / \beta\right), \xi, \alpha_{1}\right\}$. Thus,

$$
\frac{d W_{1}}{d t}+\eta_{1} W_{1} \leq \alpha+\left(R-x_{1}\right) \beta x_{2}
$$

Now applying the theorem of differential inequalities [22], we obtain

$$
0<W_{1}(t) \leq W_{1}(0) e^{-\eta_{1} t}+\frac{\alpha}{\eta_{1}}+\int\left(R-x_{1}\right) \beta x_{2} e^{-\eta_{1} t} d t
$$


as $t \rightarrow \infty, R \rightarrow x_{1}$, which gives

$$
0 \leq W_{1} \leq \frac{\alpha}{\eta_{1}}
$$

Hence the solution of the system (2.6) is bounded in $\Omega_{1}$.

We now consider the existence of possible equilibria of the system (2.6). The system of (2.6) has three feasible equilibria, namely,

(i) boundary equilibrium: $E_{1} \equiv(\alpha, 0,0, \alpha)$,

(ii) boundary equilibrium: $E_{2} \equiv\left(\gamma / \beta_{1},\left(\alpha \beta_{1}-\gamma\right) /\left(\beta-k \beta_{1}\right) \gamma, 0, \gamma / \beta_{1}\right)$. The boundary equilibrium $E_{2}$ exists if either $\gamma / \alpha<\beta_{1}<\beta / k$ or $\beta / k<\beta_{1}<\gamma / \alpha$ is satisfied, that is, growth rate of phytoplankton biomass lies between the fraction of natural death rate of phytoplankton biomass to constant supply rate of nutrient and the fraction of nutrient uptake by phytoplankton biomass to fraction of dead phytoplankton biomass,

(iii) positive interior equilibrium: $E_{3} \equiv\left(x_{1}^{*}, x_{2}^{*}, x_{3}^{*}, R^{*}\right)$, where

$$
\begin{gathered}
x_{1}^{*}=\frac{\alpha+k \gamma x_{2}^{*}}{1+\beta x_{2}^{*}}, \quad x_{3}^{*}=\frac{1}{\mu}\left[\left(\beta_{1} x_{1}^{*}-\gamma\right)\left(1+x_{2}^{*}\right)\right], \quad R^{*}=\frac{\alpha+k \gamma x_{2}^{*}}{1+\beta x_{2}^{*}}, \\
x_{2}^{*}=\frac{-s_{2} \pm \sqrt{s_{2}^{2}-4 s_{1} s_{3}}}{2 s_{1}}, \quad s_{1}=\xi_{1}, s_{2}=-\left(\mu_{1}-\left(\xi+\xi_{1}\right)\right), s_{3}=\xi .
\end{gathered}
$$

The positive interior equilibrium $E_{3}$ exists if $\mu_{1}>\xi+\xi_{1}$, that is, the growth rate of zooplankton biomass is greater than the sum of natural death rate and death due to harmful phytoplankton, and also if $x_{1}^{*}>\gamma / \beta_{1}$ means that concentration of nutrient at equilibrium is greater than the fraction of natural death rate of phytoplankton biomass to the depletion rate of nutrient uptake by phytoplankton biomass.

\section{Dynamic Behaviour and Hopf-Bifurcation}

In the previous section we observed that the system of (2.6) have three equilibria, namely, $E_{1}(\alpha, 0,0, \alpha), E_{2}\left(\gamma / \beta,\left(\alpha \beta_{1}-\gamma\right) /\left(\beta-k \beta_{1}\right) \gamma, 0, \gamma / \beta\right)$, and $E_{3}\left(x_{1}^{*}, x_{2}^{*}, x_{3}^{*}, R^{*}\right)$. We will now examine the dynamical behaviour of the system about all the feasible equilibria.

The variational matrix for the system of (2.6) evaluated at $E_{1}$ is

$$
V_{1}=\left[\begin{array}{cccc}
-1 & -(\alpha \beta-k \gamma) & 0 & 0 \\
0 & \left(\alpha \beta_{1}-\gamma\right) & 0 & 0 \\
0 & 0 & -\xi & 0 \\
\alpha_{1} & 0 & 0 & -\alpha_{1}
\end{array}\right]
$$

The eigenvalues of the characteristic equation of $V_{1}$ are $\lambda_{1}=-1, \lambda_{2}=\left(\alpha \beta_{1}-\gamma\right)$, $\lambda_{3}=-\xi$, and $\lambda_{4}=-\alpha_{1}$. It is seen from these eigenvalues that the equilibrium $E_{1}$ is locally 
asymptotically stable if $\beta_{1}<\gamma / \alpha$, which means that the growth rate of phytoplankton due to the availability of nutrient is less than the fraction of natural death rate of phytoplankton biomass to the constant input rate of nutrient.

The variational matrix for the system of (2.6) evaluated about $E_{2}$ is

$$
V_{2}=\left[\begin{array}{cccc}
\frac{-\beta_{1}(\alpha \beta-k \gamma)}{\gamma\left(\beta-k \beta_{1}\right)} & \frac{-\left(\beta-k \beta_{1}\right) \gamma}{\beta_{1}} & 0 & 0 \\
0 & 0 & \frac{-\mu\left(\alpha \beta_{1}-\gamma\right)}{\gamma\left(\beta-k \beta_{1}\right)+\left(\alpha \beta_{1}-\gamma\right)} & \frac{\beta_{1}\left(\alpha \beta_{1}-\gamma\right)}{\gamma\left(\beta-k \beta_{1}\right)} \\
0 & 0 & j_{33} & 0 \\
\alpha_{1} & 0 & 0 & -\alpha_{1}
\end{array}\right]
$$

where

$$
j_{33}=\frac{-\left[\left(\sqrt{\xi} \gamma\left(\beta-k \beta_{1}\right)-\sqrt{\xi_{1}}\left(\alpha \beta_{1}-\gamma\right)\right)^{2}+\left(\left(\sqrt{\xi}+\sqrt{\xi_{1}}\right)^{2}-\mu_{1}\right) \gamma\left(\alpha \beta_{1}-\gamma\right)\left(\beta-k \beta_{1}\right)\right]}{\left(\gamma\left(\beta-k \beta_{1}\right)+\left(\alpha \beta_{1}-\gamma\right)\right)\left(\gamma\left(\beta-k \beta_{1}\right)\right)} .
$$

The eigenvalues $\lambda_{1}, \lambda_{2}$, and $\lambda_{3}$ of the above matrix are the roots of the following cubic equation:

$$
\lambda^{3}+\left[\frac{\beta_{1}(\alpha \beta-k \gamma)}{\gamma\left(\beta-k \beta_{1}\right)}+\alpha_{1}\right] \lambda^{2}+\frac{\alpha_{1} \beta_{1}(\alpha \beta-k \gamma)}{\gamma\left(\beta-k \beta_{1}\right)} \lambda+\alpha_{1}\left(\alpha \beta_{1}-\gamma\right)=0
$$

and the fourth eigenvalue is given by $\lambda_{4}=j_{33}$.

Clearly, $\lambda_{1}, \lambda_{2}$, and $\lambda_{3}$ have negative real parts if $(\alpha \beta-k \gamma)\left(\beta-k \beta_{1}\right)>0, \beta_{1}>\gamma / \alpha$, that is, growth rate of phytoplankton biomass is greater then the fraction of natural death rate of phytoplankton biomass to constant supply rate of nutrient and $\beta_{1}(\alpha \beta-k \gamma)\left(\beta_{1}(\alpha \beta-\right.$ $\left.k \gamma)+\alpha_{1} \gamma\left(\beta-k \beta_{1}\right)\right)>\left(\beta-k \beta_{1}\right)^{2}\left(\alpha \beta_{1}-\gamma\right) \gamma^{2}$ are satisfied, the eigenvalue $\lambda_{4}$ is negative when $\xi+\xi_{1}+2 \sqrt{\xi} \sqrt{\xi_{1}}>\mu_{1}$. Thus, we can state the following theorem.

Theorem 4.1. The second boundary equilibrium $E_{2}$ is linearly asymptotically stable if

(i) $(\alpha \beta-k \gamma)\left(\beta-k \beta_{1}\right)>0$,

(ii) $\beta_{1}>\frac{\gamma}{\alpha}$,

(iii) $\xi+\xi_{1}+2 \sqrt{\xi} \sqrt{\xi_{1}}>\mu_{1}$

(iv) $\beta_{1}(\alpha \beta-k \gamma)\left(\beta_{1}(\alpha \beta-k \gamma)+\alpha_{1} \gamma\left(\beta-k \beta_{1}\right)\right)>\left(\beta-k \beta_{1}\right)^{2}\left(\alpha \beta_{1}-\gamma\right) \gamma^{2}$,

are satisfied. 
For the sake of convenience, the equilibrium points $\left(x_{1}^{*}, x_{2}^{*}, x_{3}^{*}, R^{*}\right)$ of the system is shifted to new points $\left(n_{1}, n_{2}, n_{3}, n_{4}\right)$ through transformations $n_{1}=x_{1}-x_{1}^{*}, n_{2}=x_{2}-x_{2}^{*}$, $n_{3}=x_{3}-x_{3}^{*}, n_{4}=R-R^{*}$.

In terms of the new variables, the dynamical equations (2.6) can be written as in matrix form as

$$
\dot{X}=A X+B,
$$

where $\operatorname{dot}(\cdot)$ cover $X$ denotes the derivative with respect to time. Here $A X$ is the linear part of the system and $B$ represents the nonlinear part. Moreover,

$$
\begin{gathered}
X=\left(\begin{array}{c}
n_{1} \\
n_{2} \\
n_{3} \\
n_{4}
\end{array}\right), \quad A=\left(\begin{array}{cccc}
-\left(1+\beta x_{2}^{*}\right) & -\left(\beta x_{1}^{*}-k \gamma\right) & 0 & 0 \\
0 & \frac{\mu x_{2}^{*} x_{3}^{*}}{\left(1+x_{2}^{*}\right)^{2}} & \frac{-\mu x_{2}^{*}}{1+x_{2}^{*}} & \beta_{1} x_{2}^{*} \\
0 & \left(\frac{\mu_{1}}{\left(1+x_{2}^{*}\right)^{2}}-\xi_{1}\right) x_{3}^{*} & 0 & 0 \\
0 & 0 & -\alpha_{1}
\end{array}\right), \\
\alpha_{1} \quad \begin{array}{c}
\left(\beta_{1} n_{2} n_{4}+p_{2} p_{3} p_{4}^{2} n_{2}^{3}-p_{2} p_{3} n_{2}^{2}+p_{2} p_{4} n_{2} n_{3}-p_{2} p_{4}^{2} n_{2}^{2} n_{3}+p_{2} p_{4}^{3} n_{2}^{3} n_{3}\right. \\
\left.+p_{3} p_{4} \mu n_{2}^{2}-\mu p_{4}^{2} p_{3} n_{2}^{3}-\mu p_{4} n_{2} n_{3}+\mu p_{4}^{2} n_{2}^{2} n_{3}\right)
\end{array} \\
\left(p_{3} p_{5} x_{2}^{*} n_{2}^{2}-p_{3} p_{4} p_{5} x_{2}^{*} n_{2}^{3}-p_{5} x_{2}^{*} n_{2} n_{3}+p_{4} p_{5} x_{2}^{*} n_{2}^{2} n_{3}-p_{4}^{2} p_{5} x_{2}^{*} n_{2}^{3} n_{3}\right. \\
\left.-p_{5} x_{3}^{*} n_{2}^{2}+p_{5} p_{3} n_{2}^{3}-\xi_{1} n_{2} n_{3}\right) \\
0
\end{gathered}
$$

The eigenvalues of the matrix $A$ help to understand the stability of the system. The characteristic equation for the variational matrix $A$ is given by

$$
\lambda^{4}+A_{1} \lambda^{3}+A_{2} \lambda^{2}+A_{3} \lambda+A_{4}=0
$$

where

$$
\begin{gathered}
A_{1}=p_{1}-p_{2} p_{3}, \quad A_{2}=g_{2} \alpha_{1}+p_{2} p_{5} x_{3}^{*}-p_{2}\left(p_{1} p_{3}+\xi_{1} x_{3}^{*}\right), \\
A_{3}=\alpha_{1} \beta_{1} p_{6} x_{2}^{*}+p_{1} p_{2} p_{5} x_{3}^{*}-p_{2}\left(p_{1} \xi_{1} x_{3}^{*}+g_{2} p_{3} \alpha_{1}\right), \quad A_{4}=p_{2}\left(p_{5}-\xi_{1}\right) \alpha_{1} g_{2} x_{3}^{*}, \\
p_{1}=1+\beta x_{2}^{*}+\alpha_{1}, \quad p_{2}=\frac{\mu x_{2}^{*}}{1+x_{2}^{*},} \quad p_{3}=\frac{x_{3}^{*}}{1+x_{2}^{*}, \quad p_{4}=\frac{1}{1+x_{2}^{*}} g_{2}=1+\beta x_{2}^{*},} \\
p_{5}=\frac{\mu_{1}}{\left(1+x_{2}^{*}\right)^{2}}, \quad p_{6}=\frac{\alpha \beta-k \gamma}{1+\beta x_{2}^{*}} .
\end{gathered}
$$


Using the Routh-Hurwitz criteria [21,23], we derive that the equilibrium $E_{3}$ is locally asymptotically stable, if $A_{1}>0, A_{3}>0, A_{1} A_{2}>A_{3}$, and $A_{1} A_{2} A_{3}-A_{3}^{2}-A_{1}^{2} A_{4}>0$. Here the conditions $A_{1}>0, A_{3}>0, A_{1} A_{2}>A_{3}$, and $A_{1} A_{2} A_{3}-A_{3}^{2}-A_{1}^{2} A_{4}>0$ requires

$$
\begin{gathered}
\mu_{1} p_{1}>\xi_{1} \mu x_{2}^{*} x_{3}^{*}, \quad r_{1}+r_{3}>r_{2}+r_{4}, \quad p_{1} p_{4} \alpha_{1}+L_{1}>p_{2}^{2} p_{3} p_{5} x_{3}^{*}+L_{2}, \\
r_{1} L_{1}+r_{2} L_{3}+r_{3} L_{4}+r_{4} L_{2}>r_{1} L_{3}+r_{2} L_{1}+r_{3} L_{3}+r_{4} L_{4},
\end{gathered}
$$

where $r_{1}=p_{1} p_{2} p_{5} x_{3}^{*}, r_{2}=p_{1} p_{2} \xi_{1} x_{3}^{*}, r_{3}=\alpha_{1} \beta_{1} p_{6} x_{2}^{*}, r_{4}=p_{2} p_{3} g_{2} \alpha_{1}, L_{1}=p_{1} p_{2}^{2} p_{3}+p_{2}^{2} p_{3} \xi_{1} x_{3}^{*}$, $L_{2}=p_{1}^{2} p_{2} p_{3}+\alpha_{1} \beta_{1} p_{6} x_{2}^{*}, L_{3}=L_{2}+p_{2}^{2} p_{3} p_{5} x_{3}^{*}, L_{4}=L_{1}+p_{1} g_{2} \alpha_{1}$.

Thus, we can state the following theorem.

Theorem 4.2. The interior equilibrium $E_{3}$ if it exists is linearly asymptotically stable when

$$
\begin{aligned}
& \text { (i) } \mu_{1} p_{1}>\xi_{1} \mu x_{2}^{*} x_{3}^{*}, \\
& \text { (ii) } r_{1}+r_{3}>r_{2}+r_{4}, \\
& \text { (iii) } p_{1} g_{2} \alpha_{1}+L_{1}>p_{2}^{2} p_{3} p_{5} x_{3}^{*}+L_{2}, \\
& \text { (iv) } r_{1} L_{1}+r_{2} L_{3}+r_{3} L_{4}+r_{4} L_{2}>r_{1} L_{3}+r_{2} L_{1}+r_{3} L_{3}+r_{4} L_{4} \text {, }
\end{aligned}
$$

are satisfied.

Now, we will study the Hopf-bifurcation $[24,25]$ of the system given by (2.6), taking $\alpha$ (i.e., constant input rate of nutrient) as the bifurcation parameter. The necessary and sufficient conditions for the existence of the Hopf-bifurcation for $\alpha=\alpha^{*}$, if it exists, are (i) $A_{i}\left(\alpha^{*}\right)>0$, $i=1,2,3,4$ (ii) $A_{1}\left(\alpha^{*}\right) A_{2}\left(\alpha^{*}\right)>A_{3}\left(\alpha^{*}\right)$ (iii) $A_{1}\left(\alpha^{*}\right) A_{2}\left(\alpha^{*}\right) A_{3}\left(\alpha^{*}\right)-A_{3}^{2}\left(\alpha^{*}\right)-A_{1}^{2}\left(\alpha^{*}\right) A_{4}\left(\alpha^{*}\right)=0$, and (iv) the eigenvalues of the characteristic equation (4.8) should be of the form $\lambda_{i}=u_{i}+i v_{i}$, where $d u_{i} / d \alpha \neq 0, i=1,2,3,4$. The condition $A_{1} A_{2} A_{3}-A_{3}^{2}-A_{1}^{2} A_{4}=0$ becomes

$$
-G_{1} \alpha^{3}+G_{2} \alpha^{2}+G_{3} \alpha+G_{4}=0
$$

where $G_{1}=h_{1}+f_{1}, G_{2}=h_{2}-q_{5}^{2}-f_{2}, G_{3}=h_{3}-2 q_{5} q_{6}-f_{3}, G_{4}=h_{4}-q_{6}^{2}-f_{4}, f_{1}=q_{2}^{2} q_{7}$, $f_{2}=q_{2}^{2} q_{8}-2 q_{1} q_{2} q_{7}, f_{3}=q_{1}^{2} q_{7}-2 q_{1} q_{2} q_{8}, f_{4}=q_{1}^{2} q_{8}, h_{1}=l_{2} q_{5}, h_{2}=l_{1} q_{5}-l_{2} q_{6}, h_{3}=l_{1} q_{6}+l_{3} q_{5}$, $h_{4}=l_{3} q_{6}, q_{1}=p_{1}-k_{2} g_{6}, q_{2}=k_{2} g_{5}, q_{3}=\left(p_{2} k_{6}-p_{1} k_{2}\right) g_{5}, q_{4}=\left(p_{2} k_{6}-p_{1} k_{2}\right) g_{6}+g_{2} \alpha_{1}, q_{5}=$ $\left(p_{2} k_{6} p_{1}-g_{2} \alpha_{1} k_{2}\right) g_{5}+\beta \beta_{1} \alpha_{1} g_{3} x_{2}^{*}, q_{6}=\left(p_{2} k_{6} p_{1}-g_{2} \alpha_{1} k_{2}\right) g_{6}+\left(\beta g_{4}-k \gamma\right) \beta_{1} \alpha_{1} x_{2}^{*}, q_{7}=p_{2} g_{2} \alpha_{1} k_{6} g_{5}, q_{8}=$ $p_{2} k_{6} g_{2} \alpha_{1} g_{6}, g_{1}=k \gamma x_{2}^{*}, g_{3}=1 / g_{2}, g_{4}=g_{1} / g_{2}, g_{5}=\left(1+x_{2}^{*}\right) g_{3} \beta_{1} / \mu_{1}, g_{6}=\left(1+x_{2}^{*}\right)\left(g_{4} \beta_{1}-\gamma\right) / \mu$, $k_{2}=\mu x_{2}^{*} /\left(1+x_{2}^{*}\right)^{2}, k_{6}=\mu_{1} /\left(1+x_{2}^{*}\right)^{2}-\xi_{1}$. Therefore, one pair of eigenvalues of the characteristic equation (4.8) at $\alpha=\alpha^{*}$ are of the form $\lambda_{1,2}= \pm i v$, where $v$ is positive real number.

Now, we will verify the Hopf-bifurcation condition (iv), putting $\lambda=u+i v$ in (4.8), we get

$$
(u+i v)^{4}+A_{1}(u+i v)^{3}+A_{2}(u+i v)^{2}+A_{3}(u+i v)+A_{4}=0 .
$$


On separating the real and imaginary parts and eliminating $v$ between real and imaginary parts, we have

$$
\begin{gathered}
u^{4}+v^{4}-6 u^{2} v^{2}+A_{1} u^{3}-3 A_{1} u v^{2}+A_{2}\left(u^{2}-v^{2}\right)+A_{3} u+A_{4}=0 \\
-4 u v^{3}+4 u^{3} v-A_{1} v^{3}+3 A_{1} u^{2} v+2 A_{2} u v+A_{3} v=0
\end{gathered}
$$

Substituting the value of $v^{2}$ from (4.15) in (4.14), we get

$$
\begin{aligned}
& u^{4}\left(4 u+A_{1}\right)^{2}+\left(4 u^{3}+3 A_{1} u^{2}+2 A_{2} u+A_{3}\right)^{2}+\left(4 u^{3}+3 A_{1} u^{2}+2 A_{2} u+A_{3}\right) \\
& \quad \times\left(-6 u^{2}-3 A_{1} u-A_{2}\right)\left(4 u+A_{1}\right)+\left(A_{1} u^{3}+A_{2} u^{2}+A_{3} u+A_{4}\right)\left(4 u+A_{1}\right)^{2}=0,
\end{aligned}
$$

differentiating with respect to $\alpha$ and putting $\alpha=\alpha^{*}$, we have

$$
\left[\frac{d u}{d \alpha}\right]_{\alpha=\alpha^{*}}=\frac{(-d / d \alpha)\left(A_{1} A_{2} A_{3}-A_{3}^{2}-A_{1}^{2} A_{4}\right)}{4 A_{3}\left(A_{2}-A_{3}\right)-2 A_{1}\left(A_{2}^{2}-A_{1} A_{3}\right)+8 A_{1} A_{2}} \neq 0 .
$$

Hence we can state the following theorem.

Theorem 4.3. The system (2.6) has a Hopf-bifurcation at $\alpha^{*}>0$ such that

$$
A_{1}\left(\alpha^{*}\right) A_{2}\left(\alpha^{*}\right) A_{3}\left(\alpha^{*}\right)-A_{3}^{2}\left(\alpha^{*}\right)^{2}-A_{1}^{2}\left(\alpha^{*}\right) A_{4}\left(\alpha^{*}\right)=0, \quad\left[\frac{d u}{d \alpha}\right]_{\alpha=\alpha^{*}} \neq 0 .
$$

At the Hopf-bifurcation point, the equilibrium state loses its stability and bifurcates to a periodic orbit. We obtain the value of $\alpha$ at the Hopf-bifurcation point denoted as $\alpha^{*}$ and solve the equation

$$
A_{1} A_{2} A_{3}-A_{3}^{2}-A_{1}^{2} A_{4}=0
$$

At the Hopf-bifurcation point, where the real parts of complex conjugate eigenvalues are zero, the roots of (4.8) are

$$
\lambda_{1,2}= \pm v i, \quad \lambda_{3}=-m_{1}, \quad \lambda_{4}=-m_{2}
$$

where $v=\sqrt{A_{3}} / \sqrt{A_{1}}, m_{1}=\left(-A_{1}^{2}-\sqrt{A_{1}} \sqrt{A_{1}^{3}-4 A_{1} A_{2}+4 A_{3}}\right) / 2 A_{1}$, and $m_{2}=\left(-A_{1}^{2}+\right.$ $\left.\sqrt{A_{1}} \sqrt{A_{1}^{3}-4 A_{1} A_{2}+4 A_{3}}\right) / 2 A_{1}$. 
Next, we seek a transformation matrix $P$ which reduces the matrix $A$ to the form

$$
P^{-1} A P=\left(\begin{array}{cccc}
0 & -v & 0 & 0 \\
v & 0 & 0 & 0 \\
0 & 0 & -m_{1} & 0 \\
0 & 0 & 0 & -m_{2}
\end{array}\right)
$$

where the nonsingular matrix $P$ is given as

$$
P=\left(\begin{array}{cccc}
a_{11} & a_{12} & a_{13} & a_{14} \\
0 & a_{22} & a_{23} & a_{24} \\
a_{31} & 0 & a_{31} & a_{31} \\
a_{41} & a_{42} & a_{43} & a_{44}
\end{array}\right)
$$

where, $a_{11}=v^{2} c-e d \alpha_{1}-\alpha_{1} v, a_{12}=\alpha_{1} v c+v^{3}+e d \omega, a_{13}=\left(\alpha_{1}-m_{1}\right)\left(c m_{1}+m_{1}^{2}-e d\right)$, $a_{14}=\left(\alpha_{1}-m_{2}\right)\left(c m_{2}+m_{2}^{2}-e d\right), a_{22}=\alpha_{1} v \beta_{1} x_{2}^{*}, a_{23}=\alpha_{1} m_{1} \beta_{1} x_{2}^{*}, a_{24}=\alpha_{1} m_{2} \beta_{1} x_{2}^{*}, a_{31}=$ $\alpha_{1} e \beta_{1} x_{2}^{*}, a_{41}=\left(\omega^{2}+e d\right) \alpha_{1}, a_{42}=v c \alpha_{1}, a_{43}=\left(e d-c m_{1}-m_{1}^{2}\right) \alpha_{1}, a_{44}=\left(e d-c m_{2}-m_{2}^{2}\right) \alpha_{1}$, $c=\mu x_{2}^{*} x_{3}^{*} /\left(1+x_{2}^{*}\right)^{2}, d=-\mu x_{2}^{*} /\left(1+x_{2}^{*}\right), e=\left(\mu_{1} /\left(1+x_{2}^{*}\right)^{2}-\xi_{1}\right) x_{3}^{*}$.

To achieve the normal form of (4.6), we make another change of variable, that is, $X=$ $P Y$, where $Y=\left(\begin{array}{l}y_{1} \\ y_{2} \\ y_{3} \\ y_{4}\end{array}\right)$.

Through some algebraic manipulations, (4.6) takes the form

$$
\dot{Y}=\Omega Y+F,
$$

where $\Omega=P^{-1} A P$ and

$$
F=P^{-1} f=\left(\begin{array}{l}
F^{1}\left(y_{1}, y_{2}, y_{3}, y_{4}\right) \\
F^{2}\left(y_{1}, y_{2}, y_{3}, y_{4}\right) \\
F^{3}\left(y_{1}, y_{2}, y_{3}, y_{4}\right) \\
F^{4}\left(y_{1}, y_{2}, y_{3}, y_{4}\right)
\end{array}\right), f \text { is given by } f=\left(\begin{array}{l}
f^{1}\left(y_{1}, y_{2}, y_{3}, y_{4}\right) \\
f^{2}\left(y_{1}, y_{2}, y_{3}, y_{4}\right) \\
f^{3}\left(y_{1}, y_{2}, y_{3}, y_{4}\right) \\
f^{4}\left(y_{1}, y_{2}, y_{3}, y_{4}\right)
\end{array}\right) \text {, }
$$

where, $f^{1}\left(y_{1}, y_{2}, y_{3}, y_{4}\right)=-\beta\left(a_{11} y_{1}+a_{12} y_{2}+a_{13} y_{3}+a_{14} y_{4}\right)\left(a_{22} y_{2}+a_{23} y_{3}+a_{24} y_{4}\right)$, $f^{2}\left(y_{1}, y_{2}, y_{3}, y_{4}\right)=-\mu p_{4}\left(a_{22} y_{2}+a_{23} y_{3}+a_{24} y_{4}\right)\left(a_{31} y_{1}+a_{31} y_{3}+a_{31} y_{4}\right)+\mu p_{4}^{2}\left(a_{22} y_{2}+a_{23} y_{3}+\right.$ $\left.a_{24} y_{4}\right)^{2}\left(a_{31} \mathrm{y}_{1}+a_{31} y_{3}+a_{31} y_{4}\right)-p_{2} p_{4}^{2}\left(a_{22} y_{2}+a_{23} y_{3}+a_{24} y_{4}\right)^{2}\left(a_{31} y_{1}+a_{31} y_{3}+a_{31} y_{4}\right)+p_{2} p_{4}^{3}\left(a_{22} y_{2}+\right.$ $\left.a_{23} y_{3}+a_{24} y_{4}\right)^{3}\left(a_{31} y_{1}+a_{31} y_{3}+a_{31} y_{4}\right)+\left(a_{22} y_{2}+a_{23} y_{3}+a_{24} y_{4}\right)\left(a_{41} y_{1}+a_{42} y_{2}+a_{43} y_{3}+a_{44} y_{4}\right) \beta_{1}+$ $p_{2} p_{4}\left(a_{22} y_{2}+a_{23} y_{3}+a_{24} y_{4}\right) x_{3}^{*}+\mu p_{4}^{2}\left(a_{22} y_{2}+a_{23} y_{3}+a_{24} y_{4}\right)^{2} x_{3}^{*}-p_{2} p_{4}^{2}\left(a_{22} y_{2}+a_{23} y_{3}+a_{24} y_{4}\right)^{2} x_{3}^{*}-$ $\mu p_{4}^{3}\left(a_{22} y_{2}+a_{23} y_{3}+a_{24} y_{4}\right)^{3} x_{3}^{*}+p_{2} p_{4}^{3}\left(a_{22} y_{2}+a_{23} y_{3}+a_{24} y_{4}\right)^{3} x_{3}^{*} f^{3}\left(y_{1}, y_{2}, y_{3}, y_{4}\right)=-p_{2} p_{4}\left(a_{22} y_{2}+\right.$ $\left.a_{23} y_{3}+a_{24} y_{4}\right)\left(a_{31} y_{1}+a_{31} y_{3}+a_{31} y_{4}\right)+p_{2} p_{4}^{2}\left(a_{22} y_{2}+a_{23} y_{3}+a_{24} y_{4}\right)^{2}\left(a_{31} y_{1}+a_{31} y_{3}+a_{31} y_{4}\right)-$ $\left(a_{22} y_{2}+a_{23} y_{3}+a_{24} y_{4}\right)\left(a_{31} y_{1}+a_{31} y_{3}+a_{31} y_{4}\right) \xi_{1}-p_{2} p_{4}^{3}\left(a_{22} y_{2}+a_{23} y_{3}+a_{24} y_{4}\right)^{2}\left(a_{31} y_{1}+a_{31} y_{3}+\right.$ $\left.a_{31} y_{4}\right) x_{2}^{*}-p_{2} p_{4}\left(a_{22} y_{2}+a_{23} y_{3}+a_{24} y_{4}\right)^{2} x_{3}^{*}+p_{2} p_{4}^{2}\left(a_{22} y_{2}+a_{23} y_{3}+a_{24} y_{4}\right)^{2} x_{3}^{*}+p_{2} p_{4}^{2}\left(a_{22} y_{2}+a_{23} y_{3}+\right.$ $\left.a_{24} y_{4}\right)^{3} x_{3}^{*}-p_{2} p_{4}^{3}\left(a_{22} y_{2}+a_{23} y_{3}+a_{24} y_{4}\right)^{3} x_{3}^{*}, f^{4}\left(y_{1}, y_{2}, y_{3}, y_{4}\right)=0$. 
International Journal of Differential Equations

Equation (4.23) is the normal form of (4.6) from which the stability coefficient can be computed. In (4.6), on the right hand side the first term is linear and the second one is nonlinear in $y^{\prime}$ s. For evaluating the direction of bifurcating solution, we can evaluate the following quantities at $\alpha=\alpha^{*}$ and origin

$$
\begin{aligned}
& g_{11}=\frac{1}{4}\left(\frac{\partial^{2} F^{1}}{\partial y_{1}^{2}}+\frac{\partial^{2} F^{1}}{\partial y_{2}^{2}}+i\left(\frac{\partial^{2} F^{2}}{\partial y_{1}^{2}}+\frac{\partial^{2} F^{2}}{\partial y_{2}^{2}}\right)\right) \\
& g_{02}=\frac{1}{4}\left(\frac{\partial^{2} F^{1}}{\partial y_{1}^{2}}-\frac{\partial^{2} F^{1}}{\partial y_{2}^{2}}-2 \frac{\partial^{2} F^{2}}{\partial y_{1} \partial y_{2}}+i\left(\frac{\partial^{2} F^{2}}{\partial y_{1}^{2}}-\frac{\partial^{2} F^{2}}{\partial y_{2}^{2}}+2 \frac{\partial^{2} F^{1}}{\partial y_{1} \partial y_{2}}\right)\right) \text {, } \\
& g_{20}=\frac{1}{4}\left(\frac{\partial^{2} F^{1}}{\partial y_{1}^{2}}-\frac{\partial^{2} F^{1}}{\partial y_{2}^{2}}+2 \frac{\partial^{2} F^{2}}{\partial y_{1} \partial y_{2}}+i\left(\frac{\partial^{2} F^{2}}{\partial y_{1}^{2}}-\frac{\partial^{2} F^{2}}{\partial y_{2}^{2}}-2 \frac{\partial^{2} F^{1}}{\partial y_{1} \partial y_{2}}\right)\right), \\
& G_{21}=\frac{1}{8}\left(\frac{\partial^{3} F^{1}}{\partial y_{1}^{3}}+\frac{\partial^{3} F^{2}}{\partial y_{2}^{3}}+\frac{\partial^{3} F^{2}}{\partial y_{1}^{2} \partial y_{2}}+\frac{\partial^{3} F^{1}}{\partial y_{1} \partial y_{2}^{2}}+i\left(\frac{\partial^{3} F^{2}}{\partial y_{1}^{3}}+\frac{\partial^{3} F^{1}}{\partial y_{2}^{3}}+\frac{\partial^{3} F^{1}}{\partial y_{1}^{2} \partial y_{2}}+\frac{\partial^{3} F^{2}}{\partial y_{1} \partial y_{2}^{2}}\right)\right), \\
& h_{11}^{1}=\frac{1}{4}\left(\frac{\partial^{2} F^{3}}{\partial y_{1}^{2}}+\frac{\partial^{2} F^{3}}{\partial y_{2}^{2}}\right), \quad h_{11}^{2}=\frac{1}{4}\left(\frac{\partial^{2} F^{4}}{\partial y_{1}^{2}}+\frac{\partial^{2} F^{4}}{\partial y_{2}^{2}}\right), \\
& h_{20}^{1}=\frac{1}{4}\left(\frac{\partial^{2} F^{3}}{\partial y_{1}^{2}}-\frac{\partial^{2} F^{3}}{\partial y_{2}^{2}}-2 i \frac{\partial^{2} F^{3}}{\partial y_{1} \partial y_{2}}\right), \quad h_{20}^{2}=\frac{1}{4}\left(\frac{\partial^{2} F^{4}}{\partial y_{1}^{2}}-\frac{\partial^{2} F^{4}}{\partial y_{2}^{2}}-2 i \frac{\partial^{2} F^{4}}{\partial y_{1} \partial y_{2}}\right), \\
& w_{11}^{1}=\frac{h_{11}^{1}}{m_{1}}, \quad w_{11}^{2}=\frac{h_{11}^{2}}{m_{2}}, \quad w_{20}^{1}=\frac{h_{20}^{1}}{m_{1}+2 i w}, \quad w_{20}^{2}=\frac{h_{20}^{2}}{m_{2}+2 i w}, \\
& G_{110}^{1}=\frac{1}{2}\left(\frac{\partial^{2} F^{1}}{\partial y_{1} \partial y_{3}}+\frac{\partial^{2} F^{2}}{\partial y_{2} \partial y_{3}}+i\left(\frac{\partial^{2} F^{2}}{\partial y_{1} \partial y_{3}}-\frac{\partial^{2} F^{1}}{\partial y_{2} \partial y_{3}}\right)\right) \text {, } \\
& G_{110}^{2}=\frac{1}{2}\left(\frac{\partial^{2} F^{1}}{\partial y_{1} \partial y_{4}}+\frac{\partial^{2} F^{2}}{\partial y_{2} \partial y_{4}}+i\left(\frac{\partial^{2} F^{2}}{\partial y_{1} \partial y_{4}}-\frac{\partial^{2} F^{1}}{\partial y_{2} \partial y_{4}}\right)\right) \text {, } \\
& G_{101}^{1}=\frac{1}{2}\left(\frac{\partial^{2} F^{1}}{\partial y_{1} \partial y_{3}}-\frac{\partial^{2} F^{2}}{\partial y_{2} \partial y_{3}}+i\left(\frac{\partial^{2} F^{2}}{\partial y_{1} \partial y_{3}}+\frac{\partial^{2} F^{1}}{\partial y_{2} \partial y_{3}}\right)\right) \text {, } \\
& G_{101}^{2}=\frac{1}{2}\left(\frac{\partial^{2} F^{1}}{\partial y_{1} \partial y_{4}}-\frac{\partial^{2} F^{2}}{\partial y_{2} \partial y_{4}}+i\left(\frac{\partial^{2} F^{2}}{\partial y_{1} \partial y_{4}}+\frac{\partial^{2} F^{1}}{\partial y_{2} \partial y_{4}}\right)\right) \text {, } \\
& g_{21}=G_{21}+\left(2 G_{110}^{1} w_{11}^{1}+G_{101}^{1} w_{20}^{1}+2 G_{110}^{2} w_{11}^{2}+G_{101}^{2} w_{20}^{2}\right) .
\end{aligned}
$$

Thus, we can determine $g_{11}, g_{20}, g_{02}, g_{21}$ from (4.25), (4.26), (4.27), and (4.29), respectively. Thus, we can compute the following values:

$$
\begin{gathered}
C_{1}(0)=\frac{i}{2 w}\left[g_{20} g_{11}-\left|g_{11}\right|^{2}-\frac{1}{3}\left|g_{02}\right|^{2}\right]+\frac{g_{21}}{2}, \\
\mu_{2}=\frac{-\operatorname{Re} C_{1}(0)}{\operatorname{Re} \lambda^{\prime}\left(\alpha^{*}\right)}, \quad \tau_{2}=-\frac{\left(\operatorname{Im} C_{1}(0)+\mu_{2} \operatorname{Im} \lambda^{\prime}\left(\alpha^{*}\right)\right)}{v \alpha^{*}}, \quad \beta_{2}=2 \operatorname{Re} C_{1}(0),
\end{gathered}
$$




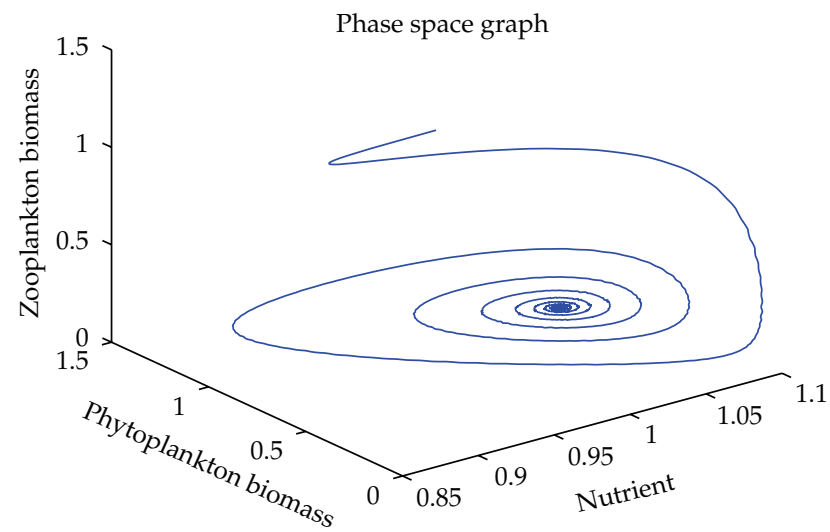

Figure 1: Phase-space diagram for nutrient, phytoplankton, and zooplankton at $\alpha=1.1$.

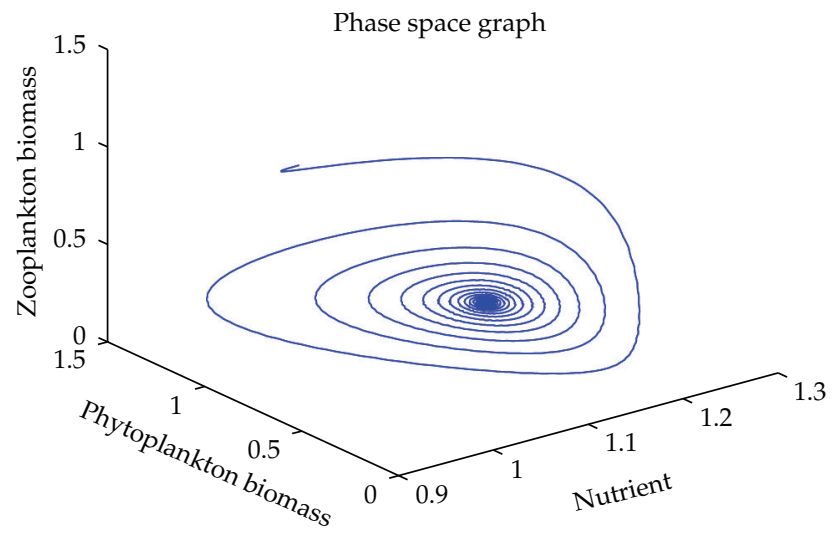

Figure 2: Phase-space diagram for nutrient, phytoplankton, and zooplankton at $\alpha=1.1775$.

which determine the qualities of bifurcation periodic solution in the center manifold at the critical value $\alpha^{*}$.

Theorem 4.4. The parameter $\mu_{2}$ determines the direction of the Hopf-bifurcation if $\mu_{2}>0\left(\mu_{2}<0\right)$, then the Hopf-bifurcation is supercritical (subcritical) and the bifurcation periodic solutions exist for $\alpha>\alpha^{*}\left(\alpha>\alpha^{*}\right) ; \beta_{2}$ determines the stability of bifurcating periodic solution; the bifurcation periodic solutions are orbitally asymptotically stable (unstable) if $\beta_{2}<0\left(\beta_{2}>0\right)$; $\tau_{2}$ determines the periodic of the bifurcating periodic solution; the period increases (decreases) if $\tau_{2}>0\left(\tau_{2}<0\right)$.

\section{Numerical Example}

In this section, we have performed numerical simulation for both systems (2.2) as well as (2.6) using MATLAB. We are taking parametric values $\beta=0.3, \gamma=0.2, k=0.3, \beta_{1}=0.25, \mu=$ $0.2, \mu_{1}=0.19, \xi=0.05, \xi_{1}=0.01$ for both systems. The behavior of the system (2.2) with different values of $\alpha$. From Figures 1, 2, 3, 4, it is observed that as $\alpha$ increases, the stable system starts oscillating. Again, with these set of values and $\alpha_{1}=0.12$, we get a positive root 


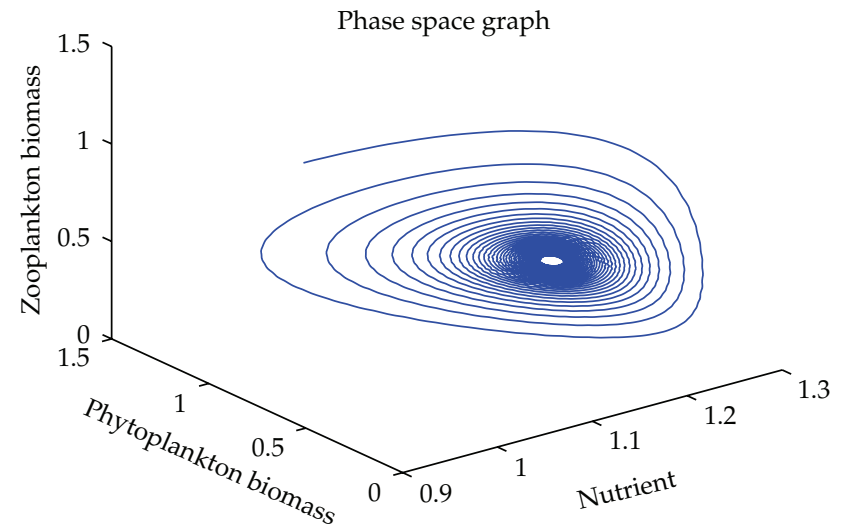

Figure 3: Stable phase-space diagram for the system with nutrient, phytoplankton, and zooplankton at $\alpha=1.25$.

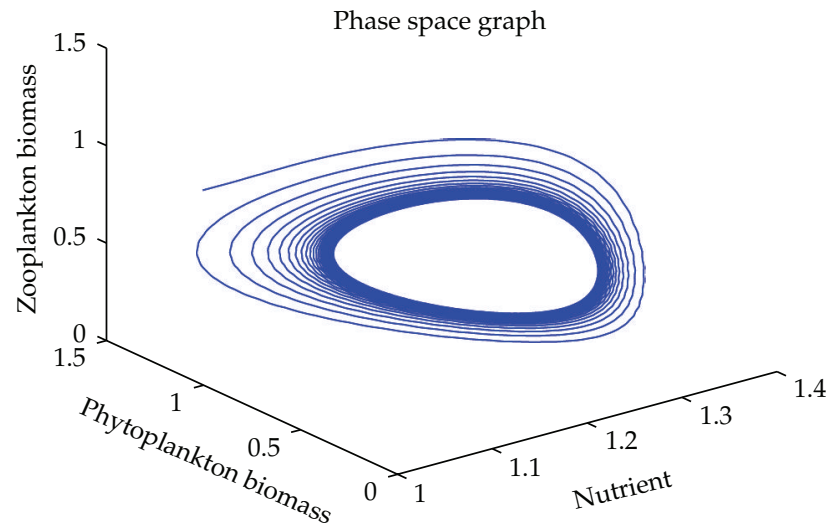

Figure 4: Phase-space diagram for nutrient, phytoplankton, and zooplankton at $\alpha=1.3$.

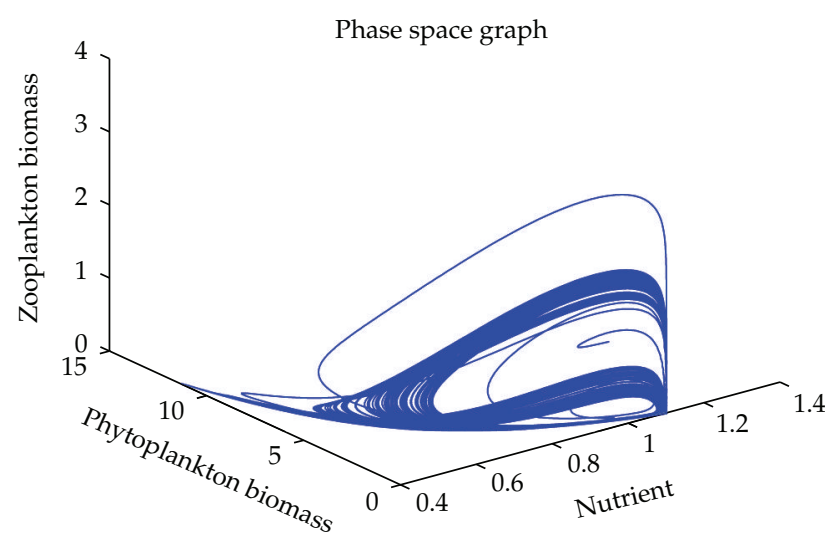

Figure 5: Phase-space diagram for nutrient, phytoplankton, and zooplankton at $\alpha=1.1, \alpha_{1}=0.01$. 


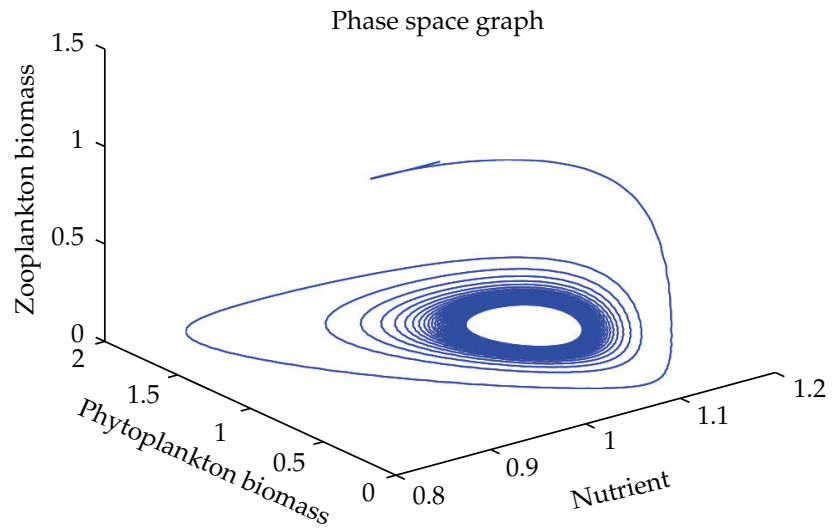

Figure 6: Phase-space diagram for nutrient, phytoplankton, and zooplankton at $\alpha=1.1, \alpha_{1}=0.07$.

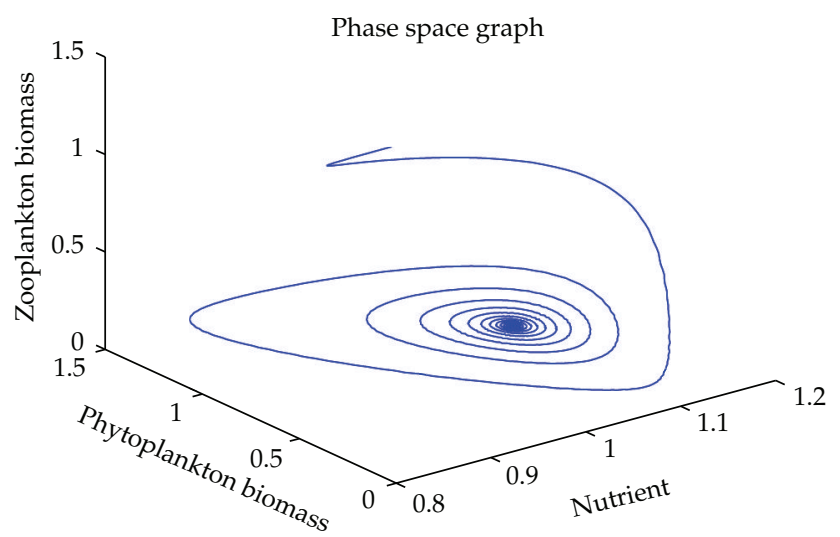

Figure 7: Stable phase-space diagram for the system with nutrient, phytoplankton, and zooplankton at $\alpha=1.1, \alpha_{1}=0.12$.

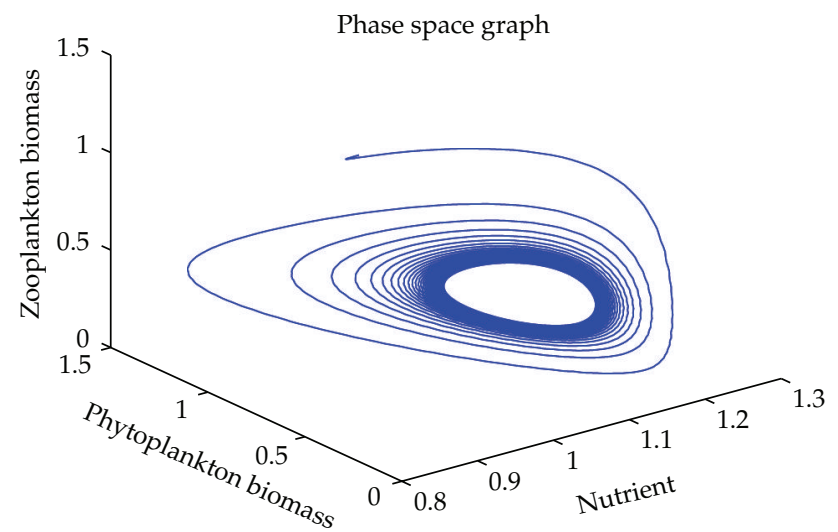

Figure 8: Phase-space diagram for nutrient, phytoplankton, and zooplankton at $\alpha=1.1775, \alpha_{1}=0.12$. 
of (4.12) is $\alpha=\alpha^{*}=1.1680$. Therefore, one pair of eigenvalues of the characteristic equation (4.8) at $\alpha=\alpha^{*}=1.1680$ are of the form $\lambda_{1,2}= \pm i v$, where $v$ is positive real number. Here the positive interior equilibrium is $E_{3}(1.06505,0.3967,0.462745)$. It follows from Section 4 , Theorem 4.3, that $\alpha^{*}=1.1680$, the positive equilibrium $E_{3}$ is stable when $\alpha<\alpha^{*}$ (see Figure 7) and the system (2.6) undergoes a Hopf-bifurcation at $\alpha>\alpha^{*}$ (see Figure 8). Now keeping $\alpha=1.1$, further reduction of $\alpha_{1}$ at 0.01 and 0.07 are shown on Figures 5-6. Moreover, we can determine the stability and direction of periodic bifurcation from the positive equilibrium at the critical point $\alpha^{*}$. For instance, when $\alpha=\alpha^{*}=1.1680, g_{11}=-0.0000105695-0.0000200596 i$, $g_{20}=0.0000362402-5.6110710^{-} 6 i, g_{02}=-0.0000151012+0.0000457302 i, g_{21}=-6.90210^{-} 8-$ $4.0204810^{-} 8 i, C_{1}(0)=-4.0826910^{-} 8+1.6292810^{-} 9 i, u^{\prime}\left(\alpha^{*}\right)=0.00115975$. It follows from (4.30) that $\mu_{2}>0$ and $\beta_{2}<0$. Therefore, the bifurcation takes place when $\alpha$ crosses $\alpha^{*}$ to the right $\left(\alpha>\alpha^{*}\right)$, and the corresponding periodic orbits are orbitally asymptotically stable, as depicted in Figure 8.

\section{Conclusion}

In this paper, we have studied the role of delay on plankton ecosystem in the presence of a toxic producing phytoplankton. In this system, it has been assumed that the toxic phytoplankton reduces the growth of zooplankton and studied the effect of delay in the digestion of nutrient by phytoplankton biomass. The local stability conditions of all the feasible equilibria of the system are established. The interior equilibrium are locally asymptotically stable under certain conditions. We have shown numerically with the set of parameters that at $\alpha_{1}=0.01$, the system exhibits the chaotic behavior (see Figure 5). Figure 6 exhibits the oscillatory behavior of the system and further it is observed that the increase in $\alpha_{1}$ makes the system stable (see Figure 7). For the comparison of the system (2.6), without delay in the system, a numerical simulation of the system (2.2) is shown in Figures $1,2,3,4$. By applying the normal form theory and the center manifold theorem, we define the explicit formulae that determine the stability and direction of the bifurcating periodic solutions. For numerical experiment, it is observed that when the input rate of nutrient, $\alpha$, exceeds the critical value $\alpha^{*}(1.1680)$, the system (2.6) leads to oscillatory behaviour shown in Figure 8 . Thus, the quantitative level of abundance of system populations depends crucially on the input rate of nutrient. Further from Theorem 4.4, we can determine the direction and stability of Hopf-bifurcation. For these chosen set of parametric values, the Hopf-bifurcation is supercritical and stable. Hence, we conclude that the supply rate $(\alpha)$ of nutrient and delay parameter $\left(\alpha_{1}\right)$ play an important role in changing the dynamical behaviour of the system.

\section{References}

[1] J. Duinker and G. Wefer, "Das $\mathrm{CO}_{2}$-problem und die rolle des ozeans,," Naturwissenschaften, vol. 81, no. 6, pp. 237-242, 1994.

[2] T. M. Work et al., "Domic acid in toxication of brown pelicans and cormorants in Santa Cruz California," in Toxic Phytoplankton Blooms in the Sea, T. J. Smayda and Y. Shimuza, Eds., vol. 3, pp. 643-649, Elsevier, Amsterdam, The Netherlands, 1993.

[3] K. A. Steidinger et al., "Pfiesteria piscicida a new toxic dinoflagellate genus and species of the order Dinamoebales," Journal of Phycology, vol. 32, pp. 157-164, 1996.

[4] T. G. Nielsen, T. Kiorboe, and P. K. Bjornsen, "Effects of a Chrysochromulina polylepis subsurface bloom on the planktonic community," Marine Ecology Progress Series, vol. 62, pp. 21-35, 1990.

[5] S. A. Levin and L. Segel, "Hypothesis for the origin plankton patchiness," Nature, vol. 259, no. 5545, article 659, 1976. 
[6] S. Uye, "Impact of copepod grazing on the red tide flagellate chattonella antiqua," Marine Biology, vol. 92, pp. 35-43, 1986.

[7] R. K. Upadhyay and J. Chattopadhyay, "Chaos to order: role of toxin producing phytoplankton in aquatic systems," Nonlinear Analysis. Modelling and Control, vol. 10, no. 4, pp. 383-396, 2005.

[8] S. R.-J. Jang, J. Baglama, and J. Rick, "Nutrient-phytoplankton-zooplankton models with a toxin," Mathematical and Computer Modelling, vol. 43, no. 1-2, pp. 105-118, 2006.

[9] T. Saha and M. Bandyopadhyay, "Dynamical analysis of toxin producing phytoplankton-zooplankton interactions," Nonlinear Analysis. Real World Applications, vol. 10, no. 1, pp. 314-332, 2009.

[10] E. J. Buskey and C. J. Hyatt, "Effects of the Texas (USA) "brown tide" alga on planktonic grazers," Marine Ecology Progress Series, vol. 126, no. 1-3, pp. 285-292, 1995.

[11] F. C. Hansen, "Trophic interactions between zooplankton and Phaeocystis cf. globosa," Helgoländer Meeresuntersuchungen, vol. 49, no. 1-4, pp. 283-293, 1995.

[12] R. Xu, Q. Gan, and Z. Ma, "Stability and bifurcation analysis on a ratio-dependent predator-prey model with time delay," Journal of Computational and Applied Mathematics, vol. 230, no. 1, pp. 187-203, 2009.

[13] N. Bairagi and D. Jana, "On the stability and Hopf bifurcation of a delay-induced predator-prey system with habitat complexity," Applied Mathematical Modelling, vol. 35, no. 7, pp. 3255-3267, 2011.

[14] S. Khare, O. P. Misra, and J. Dhar, "Role of toxin producing phytoplankton on a plankton ecosystem," Nonlinear Analysis. Hybrid Systems, vol. 4, no. 3, pp. 496-502, 2010.

[15] J. Dhar, A. K. Sharma, and S. Tegar, "The role of delay in digestion of plankton by fish population: a fishery model," Journal of Nonlinear Science and Its Applications, vol. 1, no. 1, pp. 13-19, 2008.

[16] J. Chattopadhyay, R. R. Sarkar, and A. El Abdllaoui, "A delay differential equation model on harmful algal blooms in the presence of toxic substances," IMA Journal of Mathematics Applied in Medicine and Biology, vol. 19, no. 2, pp. 137-161, 2002.

[17] N. MacDonald, Time Lags in Biological Models, vol. 27 of Lecture Notes in Biomathematics, Springer, Berlin, Germany, 1978.

[18] Y. Tang and L. Zhou, "Great time delay in a system with material cycling and delayed biomass growth," IMA Journal of Applied Mathematics, vol. 70, no. 2, pp. 191-200, 2005.

[19] Y. Tang and L. Zhou, "Stability switch and Hopf bifurcation for a diffusive prey-predator system with delay," Journal of Mathematical Analysis and Applications, vol. 334, no. 2, pp. 1290-1307, 2007.

[20] M. Kot, Elements of Mathematical Ecology, Cambridge University Press, Cambridge, UK, 2001.

[21] R. M. May, Stability and Complexity in Model Ecosystem, Princeton University Press, Princeton, NJ, USA, 2001.

[22] G. Birkhoff and G. C. Rota, Ordinary Differential Equation, Ginn, Boston, Mass, USA, 1982.

[23] J. D. Murray, Mathematical Biology, Springer, Berlin, Germany, 2001.

[24] Brian D. Hassard, N. D. Kazarinoff, and Y. H. Wan, Theory and Applications of Hopf Bifurcation, vol. 41 of London Mathematical Society Lecture Note Series, Cambridge University Press, Cambridge, UK, 1981.

[25] J. E. Marsden and M. McCracken, The Hopf Bifurcation and Its Applications, Springer, New York, NY, USA, 1976. 


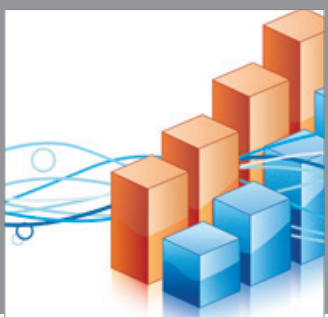

Advances in

Operations Research

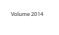

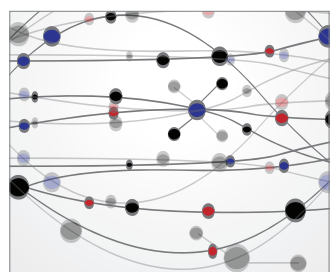

\section{The Scientific} World Journal
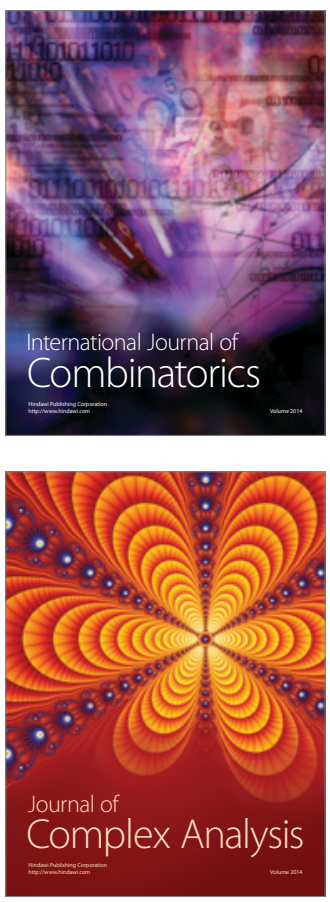

International Journal of

Mathematics and

Mathematical

Sciences
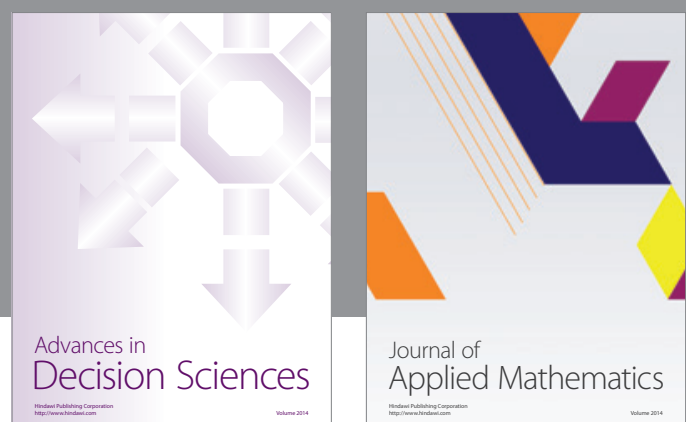

Journal of

Applied Mathematics
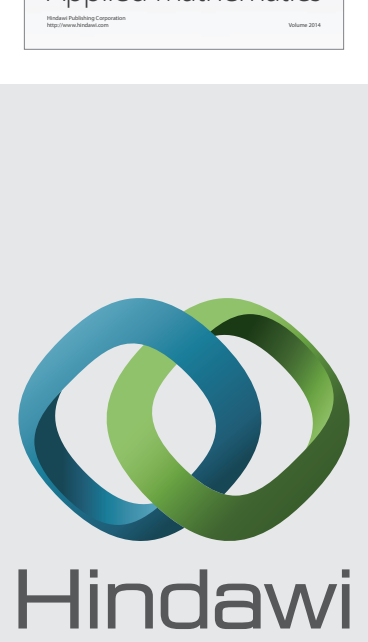

Submit your manuscripts at http://www.hindawi.com
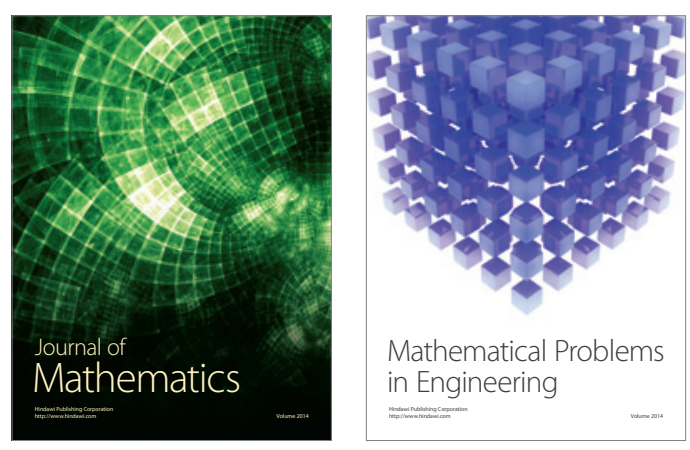

Mathematical Problems in Engineering
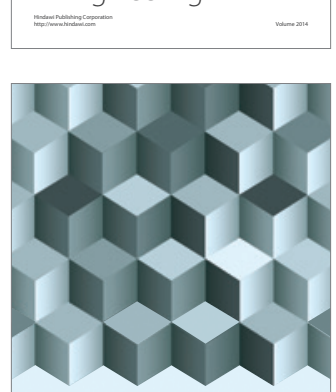

Journal of

Function Spaces
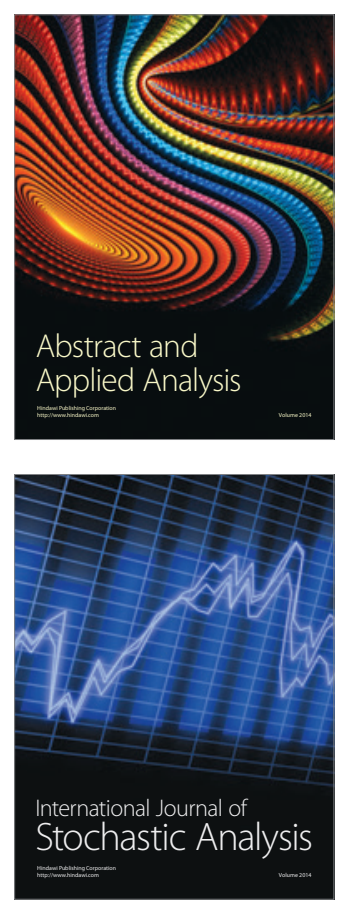

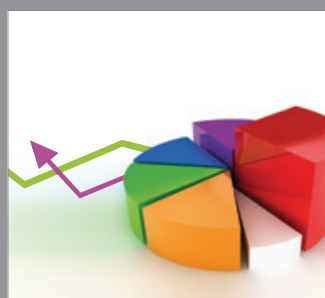

ournal of

Probability and Statistics

Promensencen
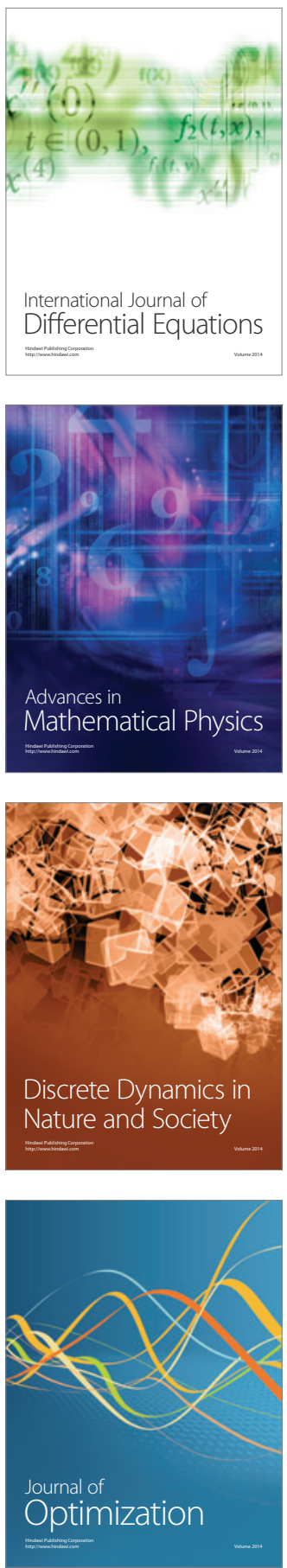